\section{Abstract A12}

Segmental Spinal Dysgenesis: Diagnostic Imaging and Neuropathology Findings in a 22 week gestational age fetus

J. Michaud, M. Valdez Quintana, S. Nikkel, D. El Demellawy, E. Miller

Children's Hospital of Eastern Ontario, University of Ottawa, Ontario, Canada

doi:10.1017/cjn.2015.378

Segmental spinal dysgenesis (SSD) is a rare congenital spinal abnormality. A 28-year-old woman was referred at 21 week gestation for a fetal MRI following sonographic findings of bilateral talipes and possible spina bifida. A prenatal diagnosis of SSD was proposed. A post-mortem fetal imaging including X-ray survey, CT and MRI was done followed by a complete autopsy. The combined major findings included: Dysplastic vertebral body below L1, absence of L2-L4 segment, presence of L5 and five sacral vertebrae, posterior dislocation of the inferior spinal segment with severe canal narrowing; thin thoraco-lumbar spinal cord above the dislocation, severe at T11 level; histological dysplasia of the thinned segment of the spinal cord with normal caudal cord; anterior intradural neurenteric cyst and segmental agenesis of the anterior spinal artery. The cranial spinal cord showed no crossing over of the cortical spinal tracts.

The above findings represent the most characteristic features of SSD. SSD should be considered in the differential diagnosis of close spinal dysraphism. It may represent a disorder of the gastrulation during the third week of gestation, a developmental step regulated by multiple genes and signaling molecules. The etiology of SSD remains uncertain. To our knowledge, this is the first case diagnosed by prenatal MRI and with a neuropathological examination.

\section{LEARNing OBJectives:}

This presentation will enable the learner

1. To define the major morphological characteristics of Segmental Spinal Dysgenesis.

2. To speculate on the pathogenesis of this rare disorder.

CONFLICTS OF INTEREST:

None.

\section{Abstract A13}

\section{Autopsy findings in pontine tegmental cap dysplasia}

Andrew Gao ${ }^{1}$, Susan Blaser ${ }^{3}$, David Chitayat ${ }^{2}$, Felice D'Arco ${ }^{3}$, Patrick Shannon ${ }^{1}$

${ }^{1}$ Depts. of Laboratory Medicine and; ${ }^{2}$ Medical Genetics, Mount Sinai Hospital; ${ }^{3}$ Dept. of Medical Imaging, Hospital for Sick Children, University of Toronto, Toronto, ON, Canada

doi:10.1017/cjn.2015.379

Pontine tegmental cap dysplasia (PTCD) is a newly described hindbrain malformation characterized by hypoplasia of the ventral pons and vaulting of the pontine tegmentum into the IVth ventricle. Approximately 20 cases have been reported but descriptions have been limited to clinical and radiologic findings. We report the first autopsy findings in PTCD.

The mother is a healthy 35-year-old G2P1 who elected to terminate pregnancy at 22 weeks gestational age due to radiologic findings of severe hindbrain abnormalities. General autopsy revealed a vertebral segmentation defect. Pertinent central nervous system findings included cerebellar hypoplasia and architectural disarray of the brainstem tegmentum. In the rostral pons, the midline tegmentum was occupied by a wedge of neurons, splaying the normal elements laterally and partially filling the ventral IVth ventricle. The nucleus basis pontis was absent, with apparent caudal displacement into the medulla. In the dorsal and caudal pons, the tegmentum was obscured by tracts of unmyelinated axons of uncertain destination running tangentially, obscuring the floor of the IVth ventricle. The inferior olivary nuclear complex was severely hypoplastic.

Our report provides the first pathologic description of PTCD and supports the radiological descriptions. In addition to the current hypothesis that PTCD represents a defect of axon guidance, our findings demonstrate abnormal neuronal migration as fundamental.

CONFLiCTS OF INTEREST:

None.

\section{Abstract A14}

\section{Neuronal Migration Abnormalities in Mitochondrial Cytochrome b Gene Mutation}

Boleslaw Lach, Janet Simons, Samantha Martin, Lauren MacNeil, Steve Sommers, Mark Tarnopolsky

McMaster University, Hamilton, Ontario

doi:10.1017/cjn.2015.380

We report two novel mDNA mutations in the cytochrome $b$ gene associated with complex III deficiency in two patients with developmental brain abnormalities.

Patient \#1. Six year old boy with history of developmental delay, myalgias, muscle weakness, high CK, Wolff-ParkinsonWhite syndrome and increased lactate in brain on MRI underwent muscle biopsy that revealed early red ragged fibers (RRF). He died in sleep at the age of 21 . Post mortem revealed accumulation of mitochondria in skeletal muscles, diaphragm and myocardium. The brain showed foci of heterotopic neurons and acellular patches of synaptophysin immunoreactivity in the white matter, and meningeal grey matter heterotopia.

Patient \#2. This microcephalic girl was prematurely born blind and deaf at the 27th gestational week. She developed seizures at six months followed by spasticity, decorticate posturing, clonus, and progressive respiratory failure. She died at the age of five. Neuronal heterotopia were present throughout the white matter. Many small vessels showed hyalinosis and subendothelial calcifications. Cerebellum was atrophic and olivary nuclei depopulated.

Although our patients had identical mDNA mutation, they displayed striking difference in the severity of manifestations as well as neuropathological abnormalities, to a large extent 